\title{
THE EXTENSION OF INTERIORITY, WITH SOME APPLICATIONS $\left.{ }^{1}\right)$
}

\author{
BY \\ C. J. TITUS AND G. S. YOUNG
}

1. Introduction. In this paper, we are interested in studying the behavior of a class of mappings on $n$-manifolds that we will call locally sense-preserving. The class is a subclass of the quasi-open maps, defined below. It includes all simplicial light interior maps, and all differentiable maps with non-negative Jacobian for which the Jacobian has rank zero whenever it is zero on an open set.

The sort of properties that we study can best be shown by listing several theorems that are special cases or corollaries of some of our results.

Theorem A. A light, locally sense-preserving map of an $n$-manifold into an n-manifold is interior, has scattered point-inverses, and is a local homeomorphism on a dense open set.

THEOREM B. Let $f: E^{n} \rightarrow E^{n}$ be a continuous map of $n$-space into $n$-space that is light and locally sense-preserving in both the open upper and lower halfspaces determined by the hyperplane $P_{n}$ given by $x_{n}=0$. Let the image of $P_{n}$ be a hyperplane. Then a necessary and sufficient condition that $f$ be light and interior on all of $E^{n}$ is that $f \mid P_{n}$ be light.

This result is a topological form of a Schwarz Reflection Principle, and is new even in $E^{2}$. Its utility is shown by the next theorem, a topological form of the classical Schwarz result.

Theorem C. Let $D$ be an open set in $E^{2}$, and let $A$ be an arc spanning $D$, such that $D-A=D_{1} \cup D_{2}$, disjoint open sets. Let $f: D \rightarrow E^{2}$ be continuous and light on $D$ and interior in $D_{1}$ and $D_{2}$. Suppose that $f(A)$ contains no open set. Then $f$ is interior and light in all of $D$.

Besides such results, we study in $\S 6$ topological results related to Cauchy's problem for elliptic equations and show that the well-known difficulties have topological reasons. The results here are too complicated to state briefly, but should apply, e.g., to function algebras.

2. Locally sense-preserving maps. A continuous function $f: A \rightarrow B$ is quasi-open provided that for any image point $q$ and any open set $U$ containing a compact component of $f^{-1}(q), q$ is in the interior of $f(U)$ relative to $B$ $[11$, p. 110]. A light quasi-open mapping is open, that is, the image of any

Received by the editors June 12, 1961.

(1) The work of the second author was supported by a National Science Foundation grant. 
open set is open in $B$. If $A$ is compact, the definition is equivalent to saying that the light factor in the monotone-light factorization of $f$ is open.

Let $M$ and $N$ be orientable $n$-manifolds. A mapping $f: M \rightarrow N$ is sensepreserving at the point $p$ of $M$ provided that if $K$ denotes the component of $f^{-1}(f(p))$ that contains $p$, then $K$ is compact and there is an open set $W$ containing $K$ such that if $U$ is any open subset of $W$ that contains $K$ but that has no point of $f^{-1}(f(p))$ on its boundary, then the degree of $f \mid U$ at $p$, $\operatorname{deg}(f \mid U, p)$, is positive. The map $f$ is locally sense-preserving if it is sensepreserving at each point.

The reason for requiring $W$ to be a neighborhood of $K$ rather than of $p$ is the same as that for requiring (Bdry $U) \cap f^{-1}(f(p))$ to be empty: $\operatorname{deg}(f \mid U, x)$ is defined only for points not in $f(\operatorname{Bdry} U)$. To require that $\operatorname{deg}(f \mid W, f(p))>0$ is not sufficient. Some of $W$ could be mapped negatively and some positively on to $f(p)$ so that $\operatorname{deg}(f \mid W, f(p))>0$, but a smaller open set than $W$ about $K$ might be mapped negatively.

The requirement that $K$ be compact is the simplest way of avoiding the difficulties that arise in the theory of degree for open sets with noncompact closure. In our case, if $K$ were not required to be compact, one could show that every open set $W$ containing $K$ also contains open sets $U$ about $K$ such that $f^{-1}(f(p)) \cap$ Bdry $U$ is empty, but such that $f$ (Bdry $U$ ) had $p$ as a limit point. Such open sets can be formed by having their boundaries approach $K$ asymptotically, and for such sets $U, \operatorname{deg}(f \mid U, f(p))$ would not be defined. Even if the requirement on $U$ were changed to requiring that $\mathrm{Cl}[f(\operatorname{Bdry} U)]$ and $f(p)$ were disjoint, it would still be true that many of the properties of degree that we use in our proofs would not hold.

We now define a class $\Omega$ of mappings $f: M \rightarrow N$, where $M$ and $N$ are fixed orientable $n$-manifolds, by the following requirements.

(i) For each function $f$ in $\Omega$ there is a closed set $C_{f}$ such that $f$ is sensepreserving at each point of $M-C_{f}$.

(ii) Each function $f$ in $\Omega$ is constant on each component of the interior of $C_{f}$.

(iii) For each function $f$ in $\Omega$, the set $f\left(C_{f}\right)$ is closed and nowhere dense in $N$.

The class $\Omega$ generalizes the class $F$ considered by Titus in [8] and the classes considered by both of us in [9].

Theorem 1. Every function in $\Omega$ is quasi-open. If $f$ is in $\Omega$ and $p$ is a point of $C_{f}$ that lies in a compact component of $f^{-1}(f(p))$, then $f$ is sense-preserving at $p$.

Proof. Let $f$ be a function in $\Omega, q$ be a point of $N, K$ be a compact component of $f^{-1}(q)$, and $U^{\prime \prime}$ be an open set containing $K$. Then $U^{\prime \prime}$ contains an open set $U^{\prime}$ containing $K$ and having a compact closure. The set $K$ is still a component of the set $f^{-1}(q) \cup B d r y U^{\prime}$, so that there is a closed set $X$ in $U^{\prime}$ such that $\bar{U}^{\prime}-X$ is the union of two relatively open sets $U$ and $V$, where $U$ 
contains $K$ and $V$ contains Bdry $U^{\prime}$ [7, Theorem 2-14, and normality]. The set $U$ is open in $M$ and has a compact closure, and $f^{-1}(q) \cap$ Bdry $U$ is empty, using the fact that Bdry $U$ is in $U^{\prime}$. If $q$ is contained in Int $f(U)$, it is contained in Int $f\left(U^{\prime \prime}\right)$, so that we need consider only such sets $U$ in proving quasi-openness.

A basic principle of the theory of degree is that if $\operatorname{deg}(f \mid U, q)$ is not zero, then the entire component $D$ of $N-f($ Bdry $U$ ) that contains $q$ is in $f(U)$. Since $D$ is open in $N$, we will be through as soon as we can show that $\operatorname{deg}(f \mid U, q)$ is positive. We show first that $q$ is a limit point of Int $f(U)$. It is well known, and easy to verify directly, that $U$ contains an open set containing $K$ that is the union of components of point-inverses. It is sufficient to let $E$ be an open set containing $q$, but such that $E \cap f$ (Bdry $U$ ) is empty; the desired open set is $f^{-1}(E) \cap U$. From this fact and from properties (i) and (ii) of the definition of $\Omega$ it follows that every open set containing $K$ contains an open set $V$ (not necessarily meeting $K$ ) such that, for some point $x$ in $V$, $\operatorname{deg}(f \mid V, f(x))>0$. It follows that Int $f(V)$ is not empty, and hence that $q$ is a limit point of Int $f(U)$. Then $D$ meets Int $f(U)$; since $f\left(C_{f}\right)$ is nowhere dense, there is a point $x$ in $D \cap f(U)$ such that each component of $f^{-1}(x)$ is in $M-C_{f}$. Each component of $f^{-1}(x)$ that lies in $U$ has a neighborhood $X$ in $U$ such that $\operatorname{deg}(f \mid X, x)>0$. It is a standard argument in degree theory that then $\operatorname{deg}(f \mid U, x)>0$. But $\operatorname{deg}(f \mid U, x)=\operatorname{deg}(f \mid U, q)$, which shows that $f$ is quasi-interior.

If $K$ lies entirely in $C_{f}$, the set $W$ of the definition of sense preserving at $p, p$ in $K$, can be taken to be the set $U$ defined above. It is easily verified that the argument above can be used to show that $f$ is sense-preserving at $p$.

The whole argument is similar to ones used in $[8 ; 9 ; 15]$. For additional details, the reader is referred to these papers.

An instructive example in connection with Theorem 1 and with the diffculties of extending our definition is the map $f: E^{2} \rightarrow E^{2}$ defined by mapping each point $(x, y)$ into the point with polar coordinates $(r, \theta)$ given by

$$
r=y, \quad \theta=\frac{2\left(x^{2}+1\right)^{1 / 2}+x}{4\left(x^{2}+1\right)^{1 / 2}} .
$$

This maps the real axis into the origin, and the upper and lower half-planes onto the region between two lines though the origin. It is locally sense-preserving away from the real axis, but the only possible value for degree at the origin would be 0 .

Even if the set $C_{f}$ is empty, it is not possible to infer that there are any totally disconnected point-inverses under the hypotheses of Theorem 1. R. D. Anderson [1] has given an example of a sense-preserving open map of the plane onto itself such that each point-inverse is a nondegenerate continuum.

TheOREM 2. Let $f$ be in $\Omega$ and let $D$ be an open set in $M-C_{f}$ such that $\bar{D}$ is 
compact. Let $q$ be a point of $N-f(\operatorname{Bdry} D)$. Then $D$ contains only a finite number of components of $f^{-1}(q)$.

Proof. The union $L$ of all components of $f^{-1}(q)$ lying in $D$ is closed, and therefore compact. Since $D$ does not meet $C_{f}$, each component $K$ of $f^{-1}(q)$ that lies in $D$ has a neighborhood $W_{k}$ satisfying with respect to $K$ the conditions put on $W$ in the definition of sense-preserving at a point. In each set $W_{k}$ there is an open set $U_{k}$ containing $K$ such that $f^{-1}(q) \cap$ Bdry $U_{k}$ is empty. A finite collection $U_{1}, U_{2}, \cdots, U_{k}$ of these sets covers $L$. Let $U_{j}$ be any one of these, and let $d_{j}=\operatorname{deg}\left(f \mid U_{j}, q\right)$. Then $d_{j}$ is positive but finite. Suppose $U_{j}$ contained more than $d_{j}$ components of $f^{-1}(q)$. Then $U_{j}$ itself contains a set of $d_{j}+1$ disjoint open sets, $U_{j 1}, U_{j 2}, \cdots, U_{j l}$, where $l=d_{j}+1$, such that (1) for each $i, 1 \leqq i<l, \operatorname{deg}\left(f \mid U_{j i}, q\right)$ is positive; (2) $L \cap U_{j}$ is contained in $\bigcup_{i-1}^{l} U_{j i}$; and (3) for each $i, 1 \leqq i \leqq l$, the set $L \cap B d r y U_{j i}$ is empty. The results on degree previously referred to imply that

$$
\operatorname{deg}\left(f \mid U_{j}, q\right)=\sum_{i=1}^{l} \operatorname{deg}\left(f \mid U_{j i}, q\right) .
$$

But this implies that

$$
\operatorname{deg}\left(f \mid U_{j}, q\right)>d_{j}
$$

a contradiction. Hence $U_{j} \cap L$ is the union of at most $d_{j}$ components of $f^{-1}(q)$, and so $L$ has at most $\sum_{j=1}^{k} d_{j}$ components.

The presence of branch points may very well reduce the actual number of components to less than $\sum d_{j}$. Consider for instance, the map $w=z^{2}$ at the origin.

We prove next a variation of Theorem 2 that is really a corollary.

THEOREM 3. Let $f$ be in $\Omega$, let $q$ be a point of $N-f\left(C_{f}\right)$, and let $D$ be an open set in $M$ with compact closure. Then only a finite number of components of $f^{-1}(q)$ meet $\bar{D}$.

Proof. The set $L^{\prime}$ of all components of $f^{-1}(q)$ that meet $\bar{D}$ is closed. For suppose that $x$ is any point of $\bar{L}^{\prime}$; then $x$ is in $f^{-1}(q)$. Let $X$ be the component of $f^{-1}(q)$ that contains $x$, and let $U$ be an open set with compact closure that contains $X$ but that has no point of $f^{-1}(q)$ on its boundary. Then every component of $f^{-1}(q)$ that meets $U$ lies in $U$. Since $U \cap L^{\prime}$ is not empty, it follows that $U \cap \bar{D}$ is not empty. But if $X \cap \bar{D}$ were empty, $U$ could be chosen not to meet $\bar{D}$.

The set $L^{\prime}$ is also countably compact, and therefore compact. For suppose that $x_{1}, x_{2}, x_{3}, \cdots$ form an infinite set of points of $L^{\prime}$ having no limit point. For each $j=1,2,3, \cdots$, let $L_{j}$ be the component of $f^{-1}(q)$ that contains $x_{j}$. Now adjoin an ideal point $\omega$ to $M$ to compactify it. Then in $M \cup \omega$, the sequence $\left\{x_{j}\right\}$ has $\omega$ for limit. It follows from [7, Theorem 2-10] that $\lim \sup L_{j}$ 
is a continuum containing $\omega$. Except for $\omega$, every point of $\lim \sup L_{j}$ is in $f^{-1}(q)$. In a continuum each component of a (relatively) open set has a closure that meets the boundary of the open set [7, Theorem 2-18]. Hence each component of $\lim \sup L_{j}-\omega$ has $\omega$ as a limit point, so is not compact in $M$. But these components are components of $f^{-1}(q)$ and under our hypotheses, these are all compact, a contradiction.

Now since $L^{\prime}$ is compact, we can find an open set containing it satisfying all the conditions on $D$ in Theorem 2, and apply Theorem 2.

3. Light interior maps. The Stoïlow-Whyburn Theorem $[11,5.1]$ states that a light interior map on a 2-manifold is locally topologically equivalent to an analytic function, and that if both the domain manifold and the image manifold are orientable the light interior map is topologically equivalent to an analytic function in the large. Thus if $f: M \rightarrow N$ is a light interior map from one orientable 2-manifold into another, then $f$ is locally sense-preserving and has scattered point-inverses. That is, for each point $x$ in $N$ each point of $f^{-1}(x)$ lies in an open set containing no other point of $f^{-1}(x)$. No such general results are known in higher dimensions, and the difficulty of the problem can be shown by the fact that if one could prove that for light interior transformations on $n$-manifolds even one point-inverse must be countable, one could give another proof of Hilbert's Fifth Problem. We can now give a few results bearing on this problem.

THEOREM 4. If $f$ is in $\Omega$ and $U$ is an open subset of $M-C_{f}$ on which $f$ is light, then $f \mid U$ has scattered inverses in $U$.

Proof. In Theorem 3, the only reason for the requirement that $q$ be a point of $N-f\left(C_{f}\right)$ is to make sure in the proof that no component of $f^{-1}(q)$ intersects both $\bar{D}$ and $C_{f}$. If $D$ is an open set such that $\bar{D}$ is a compact subset of $U$, then certainly no component of $f^{-1}(q)$ that meets $\bar{D}$ also meets $C_{f}$, so that we can infer that $f^{-1}(q) \cap \bar{D}$ is a finite set, and the theorem readily follows.

COROLLARY. If $f$ is a locally sense-preserving map of an orientable n-manifold into another one, and $f$ is light, then it is interior and has scattered point-inverses.

This result shows that there are only two ways a light interior map $f$ from an orientable $n$-manifold $M$ to an orientable $n$-manifold $N$ can fail to have scattered point-inverses. One way is for the map to be sense-reversing at some points and sense-preserving at others. The other is that there be an open subset $W$ of $M$ such that for every open subset $U$ of $W$ and for each point $x$ in $N-f($ Bdry $U), \operatorname{deg}(f \mid U, x)=0$.

4. The local homeomorphism and locally monotone properties. We now turn to a result suggested by the properties of differentiable maps in $\Omega$ whose Jacobians vanish in $C_{f}$. We prove first a preliminary result.

THEOREM 5. Let $U$ be an open set with compact closure in an n-manifold $M$. Let $f: \bar{U} \rightarrow N$ be a map of $\bar{U}$ into an n-manifold $N, f$ being continuous on $\bar{U}$ and 
locally sense-preserving on $U$. Let $q$ be a point of $N$ such that $\operatorname{deg}(f, q)=k, k \geqq 0$. Then there is a neighborhood $V$ of $q$ such that for every point $x$ in $V$, the set $f^{-1}(x)$ has no more than $k$ components.

Proof. Let the set $V$ be the component of $f(U)-f$ (Bdry $U$ ) that contains $q$. Then for each point $y$ in $V, \operatorname{deg}(f, y)=k$. Suppose that there is a point $x$ in $V$ such that $f^{-1}(x)$ has more than $k$ components. It follows easily from Theorem 3 and the compactness of $f^{-1}(x)$ that $f^{-1}(x)$ has only a finite number of components, $K_{1}, \cdots, K_{l}, l>k$. For each integer $i, i=1,2, \cdots, l$, there is an open set $V_{i}$ containing $K_{i}$ such that $f^{-1}(x) \cap$ Bdry $V_{i}$ is empty, and such that $\operatorname{deg}\left(f \mid V_{i}, x\right)>0$, since $f$ is locally sense-preserving. We may assume that the sets $V_{1}, V_{2}, \cdots, V_{l}$ are all disjoint. Then $\operatorname{deg}(f, x)=\operatorname{deg}\left(f \mid U_{i} V_{i}, x\right)$ $\geqq l>k$, a contradiction.

THEOREM 6. Let $f: M \rightarrow N$ be in $\Omega$. Let $U$ be an open set in $M$ that is the union of compact components of sets $f^{-1}(q), q$ in $N$. Then $U$ contains an open set $U^{*}$ such that (1) if $x$ is a point of $U$ that is not a limit point of $U^{*}$, then $x$ is in the interior of some component of a point-inverse; and (2) $U^{*}$ is itself the union of open sets $V$ that also are unions of components of point-inverses and that are such that $f \mid V$ is monotone.

Proof. By Theorem $1, f \mid U$ is locally sense-preserving everywhere in $U$. We also know that given an open subset $W$ of $U$ with compact closure, continuity of $f$ implies that the union of all sets in $W$ that are components of point-inverses is open in $M$. Hence if a component of a point-inverse lies in $U$, it has arbitrarily close neighborhoods that satisfy all the conditions on $U$ in the hypotheses of the theorem. It follows then that if we can show that a set $U$ satisfying the hypotheses of the theorem contains any open set $V$ that is a union of point-inverses and that is such that $f \mid V$ is monotone, our proof is completed. It also follows that we can assume that $U$ is so small that for one component $K$ of some point-inverse $f^{-1}(q)$ it is in the set $W$ of the definition of sense-preserving at $K$. With this assumption, $\operatorname{deg}(f \mid U, q)$ is a positive integer $k$.

Suppose now that $U$ contains no open set $V$ satisfying the requirements of the conclusion of the theorem. By Theorem 5 there is an open set $D$ in $N$ containing $q$ such that for each point $x$ in $D$, the set $f^{-1}(x) \cap U$ has no more than $k$ components. We will contradict this. Let $V_{1}$ be any open set in $f^{-1}(D) \cap U$ that is the union of components of point-inverses. Since $f \mid V_{1}$ is not monotone, there is some point $x_{1}$ in $D$ such that two components $K_{11}, K_{12}$ of $f^{-1}(x)$ lie in $V_{1}$. Let $W_{21}, W_{22}$ be disjoint open sets in $V_{1}$ such that $(i=1,2) W_{2 i}$ contains $K_{1 i}$ and such that each is the union of components of point-inverses. Let $V_{2 i}$ denote $f^{-1}\left[f\left(W_{21}\right) \cap f\left(W_{22}\right)\right] \cap W_{2 i}, i=1,2$. Since $f$ is quasi-interior and each component of a point-inverse in $U$ is compact, it follows that $f\left(W_{21}\right)$ and $f\left(W_{22}\right)$ are both open in $N$, and hence that $V_{21}$ and $V_{22}$ are both open in $M$; each is a union of components of point-inverses. By our denial of the theorem, 
there is some point $x_{2}$ in $f\left(W_{21}\right) \cap f\left(W_{22}\right)$ that has two components $K_{21}, K_{22}$ in $V_{21}$. Since $x_{2}$ is in $f\left(W_{22}\right), f^{-1}\left(x_{2}\right)$ certainly has a component $K_{23}$ in $V_{22}$, but conceivably only one. However, we now know that $x_{2}$ is a point whose inverse has at least three components in common with $f^{-1}(D) \cap U$. It should be clear how to proceed to get $4,5, \cdots, k+1$ components of some point-inverses in $f^{-1}(D) \cap U$, and thus find our contradiction.

Corollary 6(a). If $f: M \rightarrow N$ is a light locally sense-preserving map of a compact orientable $n$-manifold $M$ into an orientable $n$-manifold $N$, and $\operatorname{deg} f$ $=k \neq 0$, then the set of all points in $N$ whose point-inverses consist of exactly $|k|$ points is dense in $N$.

We leave the proof to the reader.

This particular corollary is a special case of a long outstanding conjecture on degree, that the corollary is true without the requirement that $f$ be locally sense-preserving. We know of no other such special case.

It is rather easy to get a map $\phi: M \rightarrow S$ from a compact manifold onto a metric space such that there is a uniform bound on the number of points in a point-inverse $\phi^{-1}(x)$, but which is not one-to-one on any open set. We know of no such example, however, where the space $S$ is a manifold of the same dimension as $M$.

Corollary 6(b). If $f$ is in $\Omega$, and is light, then there is a dense open set in $M$ on which $f$ is a local homeomorphism.

Thus the light maps in $\Omega$ share this property with light interior maps on 2-manifolds. Church and Hemmingsen [4] have recently studied this and related topics in higher dimensions.

5. An application to generalized manifolds. No one appears to have settled the question of exactly what class of generalized manifold, in the sense of Wilder [12], has a satisfactory degree and local degree theory. (A student of one of us is now considering the question for his Master's thesis.) Modulo this theory, however, our results give some information about certain generalized manifolds. Two-dimensional generalized manifolds are manifolds, but for $n \geqq 3$, a generalized manifold need not be a manifold. As an example, due essentially to Wilder, consider a monotone transformation $g: S^{3} \rightarrow A$ of the 3 -sphere onto a compact metric space $A$ such that for a dense set $\left\{x_{n}\right\}$ of points of $A, f^{-1}\left(x_{n}\right)$ is a wildly imbedded arc and such that for all other points $y, f^{-1}(y)$ is a point. By a theorem of Wilder's [13], $A$ is a generalized manifold, but it is nowhere locally Euclidean. There are a few special results for monotone transformations that imply the image is a manifold. For example, if $f: E^{3} \rightarrow A$ is a monotone map into the metric space $A$ and each point-inverse is a point or a vertical straight-line interval, then $A$ is $E^{3}$ [3]. The following theorem is the only one we know that is not of such a special nature that implies that any part of a generalized manifold is locally Euclidean. Actually, 
the hypotheses of this theorem may imply, for all we know, that the generalized manifold of its conclusion is locally Euclidean.

THEOREM 7. Let TC be a class of generalized n-manifolds containing the classical n-manifolds, for which there is a theory of degree of mappings satisfying the principal results of degree theory. Let $f: M \rightarrow N$ be a light, locally sensepreserving map of an $n$-manifold $M$ into a generalized manifold $N$ in $\mathrm{T}$. Then $N$ contains a dense open set that is locally Euclidean.

Proof. By the "principal results of degree theory" we mean those theorems required to make our previous results valid. Hence, we can use Theorem 1 to show that $f$ is interior, and can use Theorem 6 to show that there is a dense open set in $N$ that is itself the union of open sets $V$ on which $f$ is monotone. In our case, the map $f$ is then one-to-one, interior and continuous on $V$, hence a homeomorphism.

A principal difficulty in strengthening the conclusion is that we do not know whether, under a light locally sense-preserving map of $E^{3}$, it is possible for some point-inverse to be an Antoine set.

6. The Schwarz Reflection Principle. One formulation of the Schwarz Reflection Principle is the following [6, p. 184].

Theorem 8. Let $D$ be a simply connected plane domain, and let $A$ be a rectifiable arc spanning $D$, and so separating $D$ into two open sets, $D_{1}$ and $D_{2}$. Let $f: D \rightarrow E^{2}$ be continuous on $D$ and be analytic on each of $D_{1}$ and $D_{2}$. Then $f$ is analytic everywhere in $D$.

W. Rudin has kindly shown us an unpublished example of his, proving that the above result is false if $A$ is not required to be rectifiable. In Rudin's example, $A$ is an arc every subarc of which has positive 2-dimensional measure and $f$ is a space-filling curve on every subarc of $A$. It follows that $f$ cannot even be light interior in $D$, since a light interior map is topologically analytic.

Our Theorem 1 is closely related to such results. We can prove the following.

Theorem 9. Let $C$ be a closed subset of an orientable $n$-manifold $M, C$ being nowhere dense in $M$. Let $f: M \rightarrow N, N$ an orientable $n$-manifold, be light and locally sense-preserving on $M-C$, and be continuous on $M$. Suppose that $f(C)$ contains no open set. Then a necessary and sufficient condition that $f$ be interior, light, and locally sense-preserving on $M$ is that $f$ be light on $C$.

Proof. Clearly $f$ is in $\Omega$, so that by Theorem 1 , it is quasi-open and locally sense-preserving on $M$. Since it is light, it is interior on $M$.

Theorem B of the introduction is an obvious corollary. Theorem 9 is new even in the plane, and raises a question about Theorem 8. Can the requirement that $A$ be rectifiable be replaced by the weaker requirement that $f(A)$ contain no open set? It follows immediately from Theorem 9 that $f$ is light 
and interior over all $D$; hence except at an isolated set of points $f$ is a local homeomorphism. If Theorem 8 were true without the requirement that $A$ be rectifiable, but with the additional hypothesis that $f$ be a homeomorphism, we could then conclude that except at branch points of $f$ on $A, f$ would be analytic. Since these branch points would be removable discontinuities, $f$ would be analytic there. We have been able to extend Theorem 8 , however, only by weakening some and strengthening others of the hypotheses.

7. Cauchy's problem. Cauchy's problem in the theory of partial differential equations is this: Let $u, v$ be two real functions defined on the boundary of some domain $D$ in $E^{n}$, and let $L(U)=0$ be a homogeneous second-order equation defined in $\bar{D}$. Find a particular solution $U^{*}$ of the equation that agrees with $u$ on Bdry $D$ and has a normal derivative on Bdry $D$ that agrees with $v$. For elliptic equations, it is not always possible to solve Cauchy's problem even if $D, u$, and $v$ are well behaved and are near functions $u_{0}, v_{0}$ for which the problem has a solution. For elliptic equations, also, the solutions need not be continuous in the boundary data. For the case where $D$ is the upper half plane and $L$ is the Laplacian, Hadamard $[5$, p. 32] has given an example of sequences of functions $\left\{u_{n}(x)\right\},\left\{v_{n}(x)\right\}$ defined on the $x$-axis such that $\lim u_{n}=u_{0}, \lim v_{n}=v_{0}$, such that for each integer $j(j=0,1,2, \cdots)$, the Cauchy's problem with data $u_{j}, v_{j}$ has a solution $U_{j}$, but such that $\lim _{n \rightarrow \infty} U_{n}$ $\neq U_{0}$.

Our results cast some light on this situation and show that there are good topological reasons for these phenomena wholly apart from any analytical considerations.

A second-order homogeneous linear elliptic equation in a plane domain $D$ can be transformed to a system of two linear first-order equations of elliptic type, $L_{1}(U(z), V(z))=0, L_{2}(U(z), V(z))=0, z=x+i y$. A solution $U_{0}, V_{0}$ to this system gives a map $f: D \rightarrow E^{2}$ defined by $f(z)=U_{0}(z)+i V_{0}(z)$, and $f$ is either constant or light and interior in $D$ [2]. Cauchy's problem is translated into one of, given functions $u_{0}, v_{0}$ on Bdry $D$, finding functions $U_{0}, V_{0}$ in $\bar{D}$ such that on Bdry $D, U_{0}=u_{0}, V_{0}=v_{0}$. Our results above imply that the mere fact that the solutions are light and interior is enough to cause difficulty. We give two results, among many possible, that show the difficulties that arise.

Theorem 10. Let $D$ be the upper half-plane $\operatorname{Im} z>0$ in $E^{2}$. Let $G$ be a set of functions $f: \bar{D} \rightarrow E^{2}$ that are such that (1) if $f_{1}$ and $f_{2}$ are functions in $G$, then the function $f$ defined by $f(z)=f_{1}(z)-f_{2}(z)$ is light and interior in $D$ (or constant) and continuous on $\bar{D}$, and (2) $f(z)$ is real for $z$ real. For each function $f$ in $G$, let $u_{f}$ be the function on the real axis defined by $u_{f}(x)=f(x+0 \cdot i)$. Then if $f$ and $g$ are in $G$, and $[a, b]$ is an interval of the real axis, the set of points $x$ in $[a, b]$ such that $u_{f}(x)=u_{o}(x)$ has only a finite number of components.

Proof. Suppose that for some two functions $f$ and $g$ in $G$ and some interval $[a, b]$ the theorem is false. Let $\phi$ denote the function $f-g$. Then $\phi$ vanishes in 
$[a, b]$ on a set having infinitely many components. The function $\phi$ cannot be constant; thus $\phi$ is topologically equivalent in $D$ (though not necessarily in $\bar{D})$ to an analytic function and $\phi$ is either locally sense-preserving everywhere in $D$ or is locally sense-reversing everywhere in $D$. Suppose the former. Following the classical Schwarz principle, define $\phi^{*}: E^{2} \rightarrow E^{2}$ by setting $\phi^{*}(z)=\phi(z)$ if $\operatorname{Im} z>0, \phi^{*}(z)=\bar{\phi}(\bar{z})$ if $\operatorname{Im} z<0$. Then $\phi^{*}$ is in $\Omega$. But $\phi^{*}$ is constant on a bounded set with infinitely many components, which contradicts Theorem 2.

For a linear homogeneous elliptic system, the set of all solutions over the domain of the system forms a vector space. Thus Theorem 8 shows that if we have a solution to such a system in the upper half-plane, and the solution is real on the real axis, the very slightest perturbation of the function on the real axis can give boundary conditions for which Theorem 10 shows there can be no solution of the system, even though the changed conditions may be topologically suitable in the sense that there is a light interior map satisfying these conditions.

THEOREM 11. Let $D$ be the upper half-plane $\operatorname{Im} z>0$ in $E^{2}$. For each integer $j, j=0,1,2, \cdots$, let $f_{j}: \bar{D} \rightarrow E^{2}$ be a function that is continuous on $\bar{D}$, light and interior in $D$, and real on $\operatorname{Im} z=0$, and that also is not constant on any ray in the real axis. Suppose that $\left\{f_{j}\right\}$ converges to $f_{0}$, uniformly on compact subsets of $\bar{D}$. Then for each interval $[a, b]$ of the real axis there is an integer $k$ such that for each integer $j=0,1,2, \cdots$, there is a decomposition of $[a, b]$ into $k$ or less intervals on each of which $f_{j} \mid[a, b]$ is increasing or is decreasing.

Proof. Again using the Schwarz method, we can extend each map $f_{j}$ to a map $g_{j}: E^{2} \rightarrow E^{2} \cdot(j=0,1,2, \cdots)$ such that $g_{j}$ is in $\Omega$ and such that the sequence $\left\{g_{j}\right\}$ converges to $g_{0}$ uniformly on compact subsets. The components of each set $g_{j}^{-1}(w)$ consist of points or of closed intervals of the real axis. From this, and from the theorem of Moore $[10,2.3]$ that an uppersemicontinuous collection of continua none of which separates the plane defines a monotone transformation of the plane onto itself, it follows that $(j=0,1,2, \cdots)$ the map $g_{j}$ has a factorization $g_{j}=l_{j} \cdot m_{j}$, where $m_{j}$ is a closed, monotone map of the plane onto itself sending the real axis onto the real axis, and $l_{j}$ is interior and light, and maps the real axis into the real axis.

Let $a^{\prime}=m_{j}(a)$, and $b^{\prime}=m_{j}(b)$. Then $m_{j}([a, b])=\left[a^{\prime}, b^{\prime}\right]$. For each individual map $l_{j}$ it is easy to show that there is an integer $s_{j}$ such that $\left[a^{\prime}, b^{\prime}\right]$ can be decomposed into $s_{j}$ intervals on each of which $l_{j}$ is increasing or decreasing. To see this, note that each point $x$ of $\left[a^{\prime}, b^{\prime}\right]$ has a neighborhood $U_{x}$ such that $l_{j} \mid U_{x}$ is topologically equivalent to $w=z^{n}$ on $|z|<1, x$ corresponding to $z=0$. If $x$ is not a branch point, then there is an open interval $I_{x}$ of the real axis that lies in $U_{x}$ and contains $x$, and on which $l_{j}$ is increasing or is decreasing. If $x$ is a branch point, $x$ lies in an open interval $I_{x}$ of the real axis that lies in $U$, and which is the union of two subintervals joined at $x$ on each of which $l_{x}$ is increasing or is decreasing. (Possibly it is increasing on both or decreasing 
on both.) A finite number, $n$, of intervals $I_{x}$ covers $\left[a^{\prime}, b^{\prime}\right]$. Then $\left[a^{\prime}, b^{\prime}\right]$ is the union of not more than $n+1$ intervals $I_{1}, I_{2}, \cdots, I_{r_{j}}$, on each of which $l_{j}$ is increasing or is decreasing. Each set $m^{-1}\left(I_{s}\right), s=1, \cdots, r_{j}$, is an interval, and on that interval the map $l_{j} \cdot m_{j}$ is increasing or is decreasing. Since $m_{j}$ may have intervals of constancy, we cannot say that $f_{j}$ is strictly increasing or decreasing on $m^{-1}\left(I_{s}\right)$.

The difficulty of the proof, then, is to show that the set of numbers $\left\{r_{j}\right\}$ can be chosen to be bounded. To avoid making the argument verbally complicated, we will write as though all the functions $\left\{g_{j}\right\}$ were light. Otherwise we will be repeatedly forced to make statements to cover cases of pointinverses being intervals, or forced to go through a proof of the relatively obvious fact that the factors $m_{j}, l_{j}$ can be chosen so that $m_{j} \rightarrow m_{0}, l_{j} \rightarrow l_{0}$, uniformly on compact sets.

As before, each point $x$ on $[a, b]$ has a bounded neighborhood $U_{x}$ on which $g_{0} \mid \bar{U}_{x}$ is equivalent to $w=z^{n}$ on $|z|<1$ for some integer $n>0$, with $x$ being equivalent to 0 . Then $\operatorname{deg}\left(g_{0} \mid \bar{U}_{x}, g_{0}(x)\right)=n$. Since $\bar{U}_{x}$ is compact, there is an integer $J_{x}$ such that if $j>J_{x}$ and $y$ is in $\bar{U}_{x}$, then

$$
d\left(g_{j}(y), g_{0}(y)\right)<(1 / 2) d\left[g_{0}\left(\operatorname{Bdry} U_{x}\right), g_{0}(x)\right]=(1 / 2) \Delta .
$$

It follows that for $j>J_{x}$, the natural homotopy between $g_{j} \mid \bar{U}_{x}$ and $g_{0} \mid \bar{U}_{x}$ defined by $h_{j}(y, t)=t g_{0}(y)+(1-t) g_{j}(y)$ has the property that $h_{j}\left[\left(\operatorname{Bdry} U_{x}\right) \times I\right]$ does not contain $g_{0}(x)$. From this, we infer that $\operatorname{deg}\left(g_{j} \mid \bar{U}_{x}, g_{0}(x)\right)$ $=\operatorname{deg}\left(g_{0} \mid \bar{U}_{x}, g_{0}(x)\right)=n$. We can also conclude from the particular way $h$ depends on $j$ that the spherical neighborhood of $g_{0}(x)$ of radius $(1 / 2) \Delta$ contains no point of $h_{j}\left[\left(\right.\right.$ Bdry $\left.\left.U_{x}\right) \times I\right]$ for any $j>J_{x}$. Let $W$ be the spherical neighborhood of $g_{0}(x)$ of radius $(1 / 8) \Delta$, and let $V_{x}$ be the union of the sets $g_{j}^{-1}(W)$ for all $j>J_{x}$. It is easily seen that for each $j>J_{x}, g_{j}\left(V_{x}\right)$ contains $g_{0}(x)$ but contains no point of $h_{k}\left[\left(\right.\right.$ Bdry $\left.\left.U_{x}\right) \times I\right]$ for any $k>J_{x}$. Then $V_{x}$ has the property that for each $y$ in $V_{x}, \operatorname{deg}\left(g_{j} \mid \bar{U}_{x}, g_{j}(y)\right)=n$ for each $j>J_{x}$. But then there are at most $n-1$ branch points of $g_{j}$ in $V_{x}$. Let $N_{x}$ denote the number $n-1$. We can now say that in $V_{x}$ there is an open interval $I_{x}$ of the real axis that contains $x$ and that, for all $j>J_{x}$, contains no more than $N_{x}$ branch points of $g_{j}$.

A finite set, $I_{1}, \cdots, I_{k}$, of the intervals $\left\{I_{x}\right\}$ covers $[a, b]$. Let $J_{1}, \cdots$, $J_{k}, N_{1}, \cdots, N_{k}$ be the corresponding numbers $J_{x}, N_{x}$. Then for $j>\max \left(J_{1}, \cdots, J_{k}\right)$, the interval $[a, b]$ contains no more than $N=N_{1}+\cdots$ $+N_{k}$ branch points of $g_{j}$. The argument of the second paragraph of this proof shows that for $j>J$ the interval $[a, b]$ can be broken up into $N+1$ intervals on each of which $g_{j} \mid[a, b]$ is increasing or is decreasing. There are only a finite number of functions $\left\{g_{j}\right\}$ not covered by this uniform bound $N+1$. For each of these the interval $[a, b]$ can be suitably decomposed, by our second paragraph, and it is clear that our theorem is proved.

The example of Hadamard referred to above has the property that in any interval $[-a, a]$ of the real axis, the number of times that $u_{n}(x)$ is zero in that 
interval increases indefinitely with $n$. In his case $u_{n}(x) \rightarrow 0, v_{n}(x) \equiv 0$, so that the function $f_{0}(x)$ in our Theorem 11 would be the constant zero function. Thus his example does not quite fall under our theorem, but it should be clear that lack of convergence of his solutions is caused by the situation of Theorem 11. We do not know, and are inclined against it, whether there is value now in a more thorough topological study of these questions than is given in this section. One can easily find sequences of functions $\left\{u_{n}(x)\right\}$ converging on the real axis to, say, $u_{0}(x)=x$ such that each value $y$ is taken on no more than three times by any $u_{n}(x)$ and such that, by Theorem 9 , the solution $f_{n}$ to the Laplace equation with boundary conditions $\left(u_{n}, 0\right)$ does not converge to $z$.

\section{BIBLIOGRAPHY}

1. R. D. Anderson, On monotone interior mappings in the plane, Trans. Amer. Math. Soc. 73 (1952), 211-222.

2. L. Bers and L. Nirenberg, On a representation theorem for linear elliptic systems with discontinuous coefficients and its applications, Atti del Convegno internazionale a sulle Equazioni alle derivate parziali, Trieste, 1954, Edizioni Cremonese, Rome, 1955.

3. R. H. Bing, Upper semicontinuous decompositions of $E^{3}$, Ann. of Math. 65 (1957), 363374.

4. P. T. Church and E. Hemmingsen, Light open maps on n-manifolds, Duke Math. J. 27 (1960), 527-536.

5. J. Hadamard, Lectures on Cauchy's problem in linear partial differential equations, Dover, New York, 1952.

6. E. Hille, Analytic function theory, Vol. 1, Ginn and Co., Boston, Mass., 1959.

7. J. G. Hocking and G. S. Young, Topology, Addison-Wesley, Reading, Mass., 1961.

8. C. J. Titus, Sufficient conditions that a mapping be open, Proc. Amer. Math. Soc. 10 (1959), 970-973.

9. C. J. Titus and G. S. Young, A Jacobian condition for interiority, Michigan Math. J. 1 (1952), 69-72.

10. G. T. Whyburn, Analytic topology, Amer. Math. Soc. Colloq. Publ. Vol. 28, Amer. Math. Soc., New York, 1942.

11. - Topological analysis, Princeton Univ. Press, Princeton, N. J., 1958.

12. R. L. Wilder, Topology of manifolds, Amer. Math. Soc. Colloq. Publ. vol. 32, Amer. Math. Soc., New York, 1949.

13. - Monotone mappings of manifolds, Pacific J. Math. 7 (1957), 1519-1528.

14 - Monotone mappings of manifolds. II, Michigan Math. J. 5 (1958), 19-23.

15. G. S. Young, A generalization of Liouville's theorem to higher dimensions, Math. Scand. 6 (1958), 289-292.

The University of Michigan, ANN ARbor, Michigan

TUlane University, New OrLeans, Louisiana 